\title{
Use of NCCN Guidelines, Other Guidelines, and Biomarkers for Colorectal Cancer Screening
}

Christina D. Williams, PhD, MPH'a,b; William M. Grady, MD ${ }^{\mathrm{c}, \mathrm{d}}$; and Leah L. Zullig, PhD, MPHe,f

\section{Abstract}

Colorectal cancer (CRC) remains a common cancer and significant public health burden. CRC-related mortality is declining, partly due to the early detection of CRC through robust screening. NCCN has established the NCCN Guidelines for CRC Screening to help healthcare providers make appropriate screening recommendations according to the patient's risk of developing CRC. This review describes the evolution of CRC screening guidelines for average-risk individuals, discusses the role of NCCN Guidelines for CRC Screening in cancer prevention, and comments on the current and emerging use of biomarkers for CRC screening.

J Natl Compr Canc Netw 2016;14(11):1479-1485

\section{Background}

Colorectal cancer (CRC) is the third most commonly diagnosed cancer in the United States and the second leading cause of cancer-related death among men and women. In 2016, an estimated 134,490 new CRC cases and 49,190 CRC-related deaths are expected. ${ }^{1}$ Despite the considerable public health burden of CRC, incidence and mortality rates have significantly declined in the past few decades; this trend is mainly attributed to treatment innovations and increased CRC screening. ${ }^{1-3}$ Among average-risk individuals, the goals of CRC screening are 2-fold: (1) identify and remove precancerous polyps, thereby reducing CRC incidence, and (2) detect CRC at an early stage when curative therapy is most likely possible, thereby reducing CRC mortality.

From ${ }^{a}$ Cooperative Studies Program Epidemiology Center-Durham, Durham VA Medical Center, and 'Division of Medical Oncology, Duke University Medical Center, Durham, North Carolina; 'Clinical Research Division, Fred Hutchison Cancer Research Center, and dDepartment of Medicine, University of Washington, Seattle, Washington; and eCenter for Health Services Research in Primary Care, Durham VA Medical Center, and fDivision of General Internal Medicine, Duke University Medical Center, Durham, North Carolina.

Submitted May 6, 2016; accepted for publication August 31, 2016.

The authors have disclosed that they have no financial interests, arrangements, affiliations, or commercial interests with the manufacturers
Currently, $40 \%$ of CRC cases are diagnosed as localized disease, ${ }^{3}$ with a 5 -year survival rate of $90 \% .{ }^{4}$ This review describes the evolution of CRC screening guidelines for average-risk individuals, identifies predictors of adherence to these screening guidelines, discusses the role of the NCCN Clinical Practice Guidelines in Oncology (NCCN Guidelines) for CRC Screening in cancer prevention, and comments on the current and emerging use of biomarkers for CRC screening (to view the most recent version of these guidelines, visit NCCN.org).

\section{Evolution and Comparison of CRC Screening Guidelines}

Over the past 30 years, guidelines and available options for CRC screening have evolved. In the 1980s,

of any products discussed in this article or their competitors.

This work was supported by a VA Health Services Research and Development Career Development Award (CDA 13-025) (L.L.Z.). This work was also funded by the NIH awards R01CA194663, P30CA15704, and U01CA152756 (W.M.G.). The views expressed in this article are those of the authors and do not necessarily represent the views of the Department of Veterans Affairs, Duke University, or the University of Washington.

Correspondence: Christina D. Williams, PhD, MPH, Durham VA Medical Center, 508 Fulton Street (152), Durham, NC 27705.

E-mail: Christina.Williams4@va.gov 
Williams et al

for average-risk individuals aged 50 years or older, formal CRC screening guidelines focused on annual guaiac fecal occult blood test (gFOBT). Since that time, professional societies have revised their guidelines regarding the use of fecal immunochemical tests (FIT), sigmoidoscopy, barium enema, and colonoscopy, and their use as appropriate screening modalities. Recently, FIT-DNA (ie, FIT plus stool DNA) and CT colonography (CTC) have also been included as treatment modalities. ${ }^{5}$

The most sensitive screening tests for reducing CRC mortality have the ability to detect advanced serrated polyps, advanced adenomatous polyps, and cancer. Colonoscopy is one such test and, thus, may be preferred over other screening modalities that can detect cancer but not polyps (ie, stool-based tests). Because of its ability to detect and remove precancerous polyps, colonoscopy is a useful and unique tool not only in the early detection of cancer but also in cancer prevention. ${ }^{6}$

A number of organizations have established recommendations and clinical practice guidelines for CRC screening, which encompass multiple screening modalities. CRC screening guidelines have been developed by the US Preventive Services Task Force (USPSTF), ${ }^{7}$ American Cancer Society, ${ }^{8}$ American College of Gastroenterology, ${ }^{9}$ U.S. Multi-Society Task Force,${ }^{10}$ and NCCN,${ }^{4}$ among others. Although there is general consistency among the recommendations set forth by these organizations, there are also a few notable differences in guidance. Table 1 illustrates how the NCCN Guidelines for CRC
Screening compare with those of other professional societies.

The NCCN Guidelines for CRC Screening were established to help providers make appropriate CRC screening recommendations based on patients' risk of developing CRC. ${ }^{4}$ Recommended screening methods, age at which to initiate screening, and frequency of screening vary by individual risk level. For high-risk patients (ie, those with a personal history of adenomatous polyps, sessile serrated polyps, CRC, inflammatory bowel disease, or family history), the only screening method recommended is colonoscopy. ${ }^{4}$ In contrast, for average-risk patients, NCCN recommends a choice of initial screening methods, such as colonoscopy, stool-based test, or flexible sigmoidoscopy with or without a stool-based test. ${ }^{4}$ NCCN does not currently recommend screening with CTC or barium enema. ${ }^{4}$ Similarly, the USPSTF guidelines do not include barium enema due to the availability of more sensitive tests. Additionally, recommendations regarding the use of flexible sigmoidoscopy vary in 2 ways: (1) whether gFOBT and/or FIT are included, and (2) the suggested screening interval.

Recently, both USPSTF and NCCN revised aspects of their recommendations regarding CRC screening based on recently published evidence. ${ }^{2,11}$ The crux of the updated USPSTF recommendations is the absence of a preferred screening method and added emphasis on evidence that CRC screening, in general, reduces CRC mortality for asymptomatic individuals aged 50 to 75 years. ${ }^{12,13}$ The updated evidence review did not purport that one screening

Table 1. Summary of Colorectal Cancer Screening Guidelines

\begin{tabular}{|c|c|c|c|c|}
\hline & $\mathrm{NCCN}^{2}$ & USPSTF 11 & ACS/USMSTF/ACR ${ }^{6}$ & ACG $^{9}$ \\
\hline \multicolumn{5}{|l|}{ Detect cancer } \\
\hline gFOBT & Annual & Annual & Annual & Annual \\
\hline FIT & Annual & Annual & Annual & Annual \\
\hline Stool DNA (FIT-DNA) & Every 3 y & Every 1 or $3 y$ & Every 3 y & Interval uncertain \\
\hline \multicolumn{5}{|l|}{ Detect cancer and polyps } \\
\hline Flexible sigmoidoscopy & $\begin{array}{l}\text { Every } 5-10 \text { y } \pm \\
\text { gFOBT/FIT at y } 3\end{array}$ & $\begin{array}{l}\text { Every } 5 \text { y OR } \\
\text { Every } 10 y+\text { annual FIT }\end{array}$ & $\begin{array}{l}\text { Every } 5 \mathrm{y} \pm \\
\text { annual gFOBT/FIT }\end{array}$ & Every 5 y \\
\hline Colonoscopy & Every $10 \mathrm{y}$ & Every $10 \mathrm{y}$ & Every 10 y & Every $10 \mathrm{y}$ \\
\hline CTC & - & - & Every 5 y & Every 5 y \\
\hline Barium enema & _- & _- & Every $5 y$ & Every $5 y$ \\
\hline
\end{tabular}

Abbreviations: ACG, American College of Gastroenterology; ACR, American College of Radiology; ACS, American Cancer Society; CTC, CT colonography; FIT, fecal immunochemical test; gFOBT, guaiac fecal occult blood test; USMSTF, U.S. Multi-Society Task Force; USPSTF, US Preventive Services Task Force. 
method was superior to another as it relates to the net benefit. ${ }^{7}$ The current list of screening options now includes FIT-DNA every 1 or 3 years, CTC every 5 years, and flexible sigmoidoscopy every 5 years or every 10 years with annual FIT. The USPSTF's current guidance further highlights that although this mortality benefit is greatest for those aged 50 to 75 years, the benefit for older patients is minimal. Therefore, the decision to screen should take into consideration the health and screening history for those aged 76 to 85 years, with no screening recommended for those older than 85 years. Because screening rates are suboptimal, with at least onethird of eligible patients having never been screened, the USPSTF asserts that the "best screening test is the one that gets done." Shared decision-making is an approach to choosing the "best" and preferred test for each patient, and it has been shown that patients are more likely to adhere to screening when given a choice of screening method. ${ }^{14}$

A key modification to NCCN Guidelines for average-risk individuals is the emphasis that screening not only lowers CRC mortality, but also reduces incidence by identifying and removing polyps. ${ }^{2}$ Previously, the recommended interval for flexible sigmoidoscopy was every 5 years with or without stool-based testing every 3 years, whereas current recommendations call for flexible sigmoidoscopy every 5 to 10 years with or without gFOBT/FIT at year 3. NCCN Guidelines now recommend the option of stool DNA for CRC detection with a suggested interval of 3 years, although the appropriateness of this interval is uncertain. ${ }^{15}$ Regarding stool-based screening in general, the guidelines note that evidence showing FIT is more sensitive than gFOBT was based solely on nonrandomized studies, which also indicate lower mortality with FIT. Language referencing colonoscopy as the "primary method" for CRC screening was modified to "most common method," presumably to remove the suggestion that colonoscopy is preferred over other screening modalities.

\section{Rates and Predictors of Adherence to Guidelines for Initial CRC Screening}

Given the National Colorectal Cancer Roundtable's goal to achieve CRC screening compliance rates of $80 \%$ by 2018 , it is critical to assess current rates and identify predictors of screening adherence. ${ }^{16}$ Studies have shown that older patients and, to a lesser extent, those who have a higher comorbidity burden, ${ }^{17}$ are uninsured, ${ }^{18}$ lack a high school education, are of certain race/ethnic groups, and report certain health beliefs are less likely to undergo CRC screening. ${ }^{19}$

A recent 10-year longitudinal study evaluated adherence to USPSTF guidelines and determined that only $64 \%$ of insured patients in their cohort were screened in accordance with the guidelines. Of those screened, screening was initiated an average of 3 years later than recommended..$^{20}$ A separate study at a Veterans Affairs (VA) medical center evaluated the impact of a national VA CRC screening initiative by comparing screening rates and outcomes before and after the 1998 initiative. ${ }^{21}$ The screening rates before and after implementation of the initiative were $45 \%$ and 50\%, respectively. Pre- and post-initiative stage distribution was only suggestive of a trend toward early-stage diagnoses. However, when considering anatomic location, there was a significant shift toward early-stage diagnoses among distal cancers after the initiative, but no change in stage distribution for proximal cancers. Among all patients with screen-detected CRC, the 5-year allcause survival rate was approximately $89 \%$.

In 2010, the prevalence estimate of screening by either FOBT or endoscopy (ie, sigmoidoscopy or colonoscopy) was $59 \%{ }^{3}$ Screening rates vary by a number of demographic and socioeconomic factors, including patient age, race, years of education, insurance status, and immigration status. ${ }^{3}$ In a study by Shapiro et $\mathrm{al}^{22}$ using National Health Interview Survey data, the prevalence of guideline-concordant self-reported CRC screening by any means was $58 \%$, with the use of colonoscopy being 55\%. Substantial differences in receipt of screening were noted within categories of income, education, type of insurance, and source of healthcare. The study authors also captured the patient reasons for deciding not to undergo CRC screening, with the most commonly reported reason being "no reason or never thought about it" (41\%), followed by a lack of provider recommendation (15\%) and absence of symptoms (14\%).22 A retrospective cohort study of screening-eligible patients in a safety-net health system reported 22\% of patients being screened in the prior 5 years, with significant predictors of screening participation being female sex, Hispanic ethnicity, age of 65 to 75 years, and having access to care. ${ }^{23}$ Marital status is 
Williams et al

another important predictor of screening, according to a large study of approximately 300,000 participants that noted that, compared with nonpartnered people, married and unmarried couples were more likely to undergo CRC screening. ${ }^{24}$

\section{Current and Emerging Use of Biomarkers for CRC Screening}

Despite a strong evidence base supporting its efficacy, colonoscopy is limited by its invasiveness, expense, and suboptimal patient compliance, whereas FOBT and FIT have low sensitivities for detecting colon polyps. Thus, efforts are underway to develop more sensitive and specific noninvasive biomarker assays as another means of risk stratification and early detection of advanced colon polyps or CRC. As part of the colorectal carcinogenesis process, gene mutations and epigenetic alterations arise in colon polyps and CRCs, and are potentially highly specific diagnostic biomarkers for the detection of colonic polyps and cancers. Feces and blood are the analytes used in the best developed biomarkers for CRC screening at this time, and they are the analytes used in the recently FDA-approved CRC biomarkers.

\section{Fecal-Based Biomarkers}

Since the discovery of mutant KRAS in fecal specimens of patients with CRC, ${ }^{25}$ numerous studies have supported using fecal DNA for potential screening assays for early CRC detection. ${ }^{26-28}$ To date, biomarkers assessed include mutant genes, methylated genes, microRNAs, and mRNA. However, to date, the most successful biomarkers are based on methylated DNA.

Many phase I and II biomarker studies have identified fecal-based methylation biomarkers for the early detection of CRC. ${ }^{29}$ Studies of methylated SFRP2, SFRP5, PGR, CALCA, and IGFBP2 in fecal DNA identified methylated SFRP2 as a diagnostic biomarker for CRC detection with high sensitivity $(77 \%-90 \%)$ and specificity $(77 \%) .{ }^{30}$ A second study of stool DNA markers among patients with colorectal adenomas demonstrated that methylated SFRP2 can also identify patients with precancerous colonic polyps. ${ }^{31}$ Another well-studied fecal DNA biomarker for CRC detection is methylated VIM, the gene for vimentin. Methylated VIM specifically occurs in adenoma and CRC tissues, and is detected in the fecal DNA of patients with colon neoplasms with reasonably high sensitivity $(46 \%)$ and specificity (90\%)..$^{32}$ Studies demonstrating the potential of using methylated VIM as a biomarker for the early detection of CRC has led to the development of an assay that detects methylated VIM and is one of the first commercial fecal-DNA screening tests for CRC (ColoSure, Lab Corp, Burlington, NC). Other hypermethylated genes (APC, ATM, BMP3, CDKN2A, SFRP2, GATA4, GSTP1, HLTF, MLH1, MGMT, NDRG4, RASSF2A, TFPI2, VIM, and WIF1) have been analyzed in fecal DNA for the early detection of CRC. ${ }^{26,32-40}$

Efforts to develop an accurate noninvasive biomarker assay for CRC and colon polyp detection recently culminated in the development of Cologuard (Exact Sciences Corporation, Madison, WI), an FDA-approved, clinically available stool-based CRC screening test that combines both the stool DNA test and the OC-Auto FIT (Polymedco CDP, LLC, Cortlandt Manor, NY). This stool DNA-based assay, which detects methylated BMP3, methylated NDRG4, and mutant KRAS, was recently compared with FIT and demonstrated better sensitivity than FIT for both CRC and advanced adenomas, but lower specificity for both end points. ${ }^{27,41}$ The Cologuard assay is approved for use by the FDA and in the European Union; however, its appropriate use in clinical care is in evolution. Of note, the Cologuard assay has been included as a screening method in the 2016 USPSTF screening guidelines and the 2014 American Cancer Society's CRC prevention and early detection guidelines.

\section{Blood-Based Biomarkers}

Because of accessibility and high patient acceptance, blood is invariably the most ideal analyte for cancer biomarkers. With the development of highly sensitive detection methods, such as massively parallel sequencing, there have been renewed efforts to determine whether blood-based diagnostic assays based on circulating methylated DNA can be used for detection of colon polyps or CRC. Okugawa et $\mathrm{al}^{26}$ recently summarized aberrantly methylated genes discovered in the plasma or serum of patients with CRC which, consequently, are candidate biomarkers.

After initial reports of methylated CDKN2A in circulating DNA of patients with a variety of cancers in 1999, ${ }^{42-44}$ a growing number of studies have examined the potential of methylated genes to be 
blood-based biomarkers for patients with CRC. ${ }^{26,27}$ Currently, the most established methylated DNA blood biomarker is methylated septin 9 (SEPT9). Lofton-Day et $\mathrm{al}^{45}$ identified methylated SEPT9 as a noninvasive diagnostic biomarker for CRC with $69 \%$ sensitivity and $86 \%$ specificity for distinguishing patients with CRC from healthy individuals. Upon further refinement and validation of assays that use methylated SEPT9 as a biomarker for CRC screening, it is now offered commercially as a blood-based screening test in various assays including Epi proColon 1.0 (Epigenomics, Seattle, WA), ColoVantage (Quest Diagnostics, Madison, NJ) and RealTime mS9 (Abbott Laboratories, Des Plaines, IL). A recent prospective clinical trial (PRESEPT) demonstrated equivalent sensitivity and specificity of methylated SEPT9 versus FOBT for CRC, confirming its potential usefulness as a blood-based biomarker for CRC. ${ }^{46}$ This test was recently approved by the FDA for use as a CRC screening assay. However, methylated SEPT9 has a limited sensitivity for the detection of advanced adenomas (11\%), underscoring the need for further improvement of this test for implementation for population-based screening of colorectal neoplasia. A recent study demonstrated that the methylated SEPT9 assay was superior to FIT at detecting CRC, but both approaches were suboptimal for diagnosing patients with advanced adenomas. ${ }^{47}$ To date, several other blood-based diagnostic methylation biomarkers have been identified for CRC detection, including ALX4, ${ }^{48}$ APC, ${ }^{35}$ CDKN2A, ${ }^{39} \mathrm{HLTF}^{49} \mathrm{HPP} 1,{ }^{50}$ hMLH1, ${ }^{49}$ MGMT, ${ }^{35}$ NEUROG1, ${ }^{51}$ NGFR, RASSF2A, ${ }^{35}$ SFRP2, VIM, ${ }^{52}$ and WIF1. ${ }^{35}$ Unfortunately, there are no phase III biomarker studies of mutant or methylated DNA-based biomarker assays for CRC detection in a screening setting at this time. ${ }^{53}$ Furthermore, and importantly, when considered in the context of other CRC and colon polyp screening methods, although a robust biomarker panel of methylated genes may be developed into a clinically accurate CRC screening method in the near future, the currently available blood-based biomarker assays are not appropriate as colon polyp detection methods.

\section{Future Directions}

Given the consensus that CRC screening is beneficial yet underutilized, efforts to continue to improve screening rates will expand and will likely focus on historically underscreened populations. Therefore, having a clear, and ideally unified, set of guidelines could assist in this endeavor. These unified guidelines should be accompanied by research documenting screening adherence, improvements in health outcomes, and whether screening capacity is adequate to achieve current benchmarks. Despite their limitations, blood-based tests have the potential to increase screening rates because they are noninvasive. Moreover, blood-based tests are relatively simple to conduct and may be more acceptable to patients than more invasive and labor-intensive options. Additionally, shared decision-making could result in choosing a screening option that is most consistent with patients' values and preferences, thus improving adherence for initial and, if necessary, follow-up testing. Additional interventions to improve initial CRC screening may include screening programs targeting screening-naïve and historically underscreened populations, improved care coordination, and use of patient and provider reminder tools. As CRC screening recommendations continue to evolve and new technologies and biomarkers emerge, there may be heightened interest in measuring adherence to clinical practice guidelines as a quality indicator. Systematic monitoring of screening and associated health outcomes must be developed and implemented.

\section{Conclusions}

Although CRC screening rates have increased dramatically over the past few decades, they remain suboptimal. Reasons for underutilization of CRC screening methods are multifactorial and accordingly require changes at multiple levels, including the patient, provider, and healthcare system. The guidelines developed by numerous professional organizations continue to evolve as more evidence becomes available regarding the sensitivity, specificity, benefits, harms, cost, and availability of current and emerging screening options. There is variation between guidelines regarding which screening methods are endorsed and the associated time intervals for screening; the screening intervals for FIT-DNA and flexible sigmoidoscopy are the least consistent across guidelines. The revised guidelines should focus less on the specific screening methods provided to patients and instead emphasize the importance of increasing CRC screening rates overall. Although unified guidance for CRC 
screening may not be on the immediate horizon, improving uptake of CRC screening and, consequently, improving early detection and reducing mortality is something that all societies support.

\section{References}

1. American Cancer Society. Cancer Facts \& Figures 2016. Available at: http:// www.cancer.org/acs/groups/content/@research/documents/document/ acspc-047079.pdf. Accessed July 28, 2016.

2. Provenzale D, Gupta S, Ahnen DJ, et al. Colorectal Cancer Screening, version 1.2016. National Comprehensive Cancer Network. Accessed July 27,2016 . To view the most recent version of these guidelines, visit NCCN. org.

3. Siegel R, Desantis C, Jemal A. Colorectal cancer statistics, 2014. CA Cancer J Clin 2014;64:104-117.

4. Provenzale D, Jasperson K, Ahnen DJ, et al. Colorectal Cancer Screening, version 1.2015. J Natl Compr Canc Netw 2015;13:959-968; quiz 968.

5. Jensen CD, Corley DA, Quinn VP, et al. Fecal immunochemical test program performance over 4 rounds of annual screening: a retrospective cohort study. Ann Intern Med 2016;164:456-463.

6. Levin B, Lieberman DA, McFarland B, et al. Screening and surveillance for the early detection of colorectal cancer and adenomatous polyps, 2008: a joint guideline from the American Cancer Society, the US Multi-Society Task Force on Colorectal Cancer, and the American College of Radiology. Gastroenterology 2008;134:1570-1595.

7. Whitlock EP, Lin JS, Liles E, et al. Screening for colorectal cancer: a targeted, updated systematic review for the U.S. Preventive Services Task Force. Ann Intern Med 2008;149:638-658.

8. Byers $T$, Levin B, Rothenberger D, et al. American Cancer Society guidelines for screening and surveillance for early detection of colorectal polyps and cancer: update 1997. American Cancer Society Detection and Treatment Advisory Group on Colorectal Cancer. CA Cancer J Clin 1997;47:154-160.

9. Rex DK, Johnson DA, Anderson JC, et al. American College of Gastroenterology guidelines for colorectal cancer screening 2008. Am J Gastroenterol 2009;104:739-750.

10. Lieberman DA, Rex DK, Winawer SJ, et al. Guidelines for colonoscopy surveillance after screening and polypectomy: a consensus update by the US Multi-Society Task Force on Colorectal Cancer. Gastroenterology 2012;143:844-857.

11. Lin JS, Piper MA, Perdue LA, et al. Screening for colorectal cancer: updated evidence report and systematic review for the US Preventive Services Task Force. JAMA 2016;315:2576-2594.

12. Bibbins-Domingo K, Grossman DC, Curry SJ, et al. Screening for colorectal cancer: US Preventive Services Task Force recommendation statement. JAMA 2016;315:2564-2575.

13. Ransohoff DF, Sox HC. Clinical practice guidelines for colorectal cancer screening: new recommendations and new challenges. JAMA 2016;315:2529-2531.

14. Inadomi JM, Vijan $S$, Janz $\mathrm{NK}$, et al. Adherence to colorectal cancer screening: a randomized clinical trial of competing strategies. Arch Inter Med 2012;172:575-582.

15. Berger BM, Parton MA, Levin B. USPSTF colorectal cancer screening guidelines: an extended look at multi-year interval testing. Am J Manag Care 2016;22:e77-81.

16. Meester RG, Doubeni CA, Zauber AG, et al. Public health impact of achieving $80 \%$ colorectal cancer screening rates in the United States by 2018. Cancer 2015;121:2281-2285.

17. Klabunde CN, Zheng Y, Quinn VP, et al. Influence of age and comorbidity on colorectal cancer screening in the elderly. Am J Prev Med 2016;51:e6775

18. Joseph DA, King JB, Miller JW, Richardson LC. Prevalence of colorectal cancer screening among adults-behavioral risk factor surveillance system, United States, 2010. MMWR Suppl 2012;61:51-56.

19. Stanley SL, King JB, Thomas CC, Richardson LC. Factors associated with never being screened for colorectal cancer. J Community Health 2013;38:31-39.

20. Cyhaniuk A, Coombes ME. Longitudinal adherence to colorectal cancer screening guidelines. Am J Manag Care 2016;22:105-111.
21. van Roessel P, Rouse RV, Wren SM. Care within a Veterans Hospital earlier detection of colon cancer. Surg Endosc 2007;21:1434-1440.

22. Shapiro JA, Klabunde CN, Thompson TD, et al. Patterns of colorectal cancer test use, including CT colonography, in the 2010 National Health Interview Survey. Cancer Epidemiol Biomarkers Prev 2012;21:895-904.

23. Gupta S, Tong LY, Allison JE, et al. Screening for colorectal cancer in a safety-net health care system: access to care is critical and has implications for screening policy. Cancer Epidemiol Biomarkers Prev 2009;18:23732379.

24. El-Haddad B, Dong F, Kallail KJ, et al. Association of marital status and colorectal cancer screening participation in the USA. Colorectal Dis 2015;17:O108-114

25. Sidransky D, Tokino T, Hamilton SR, et al. Identification of ras oncogene mutations in the stool of patients with curable colorectal tumors. Science 1992;256:102-105.

26. Okugawa Y, Grady WM, Goel A. Epigenetic alterations in colorectal cancer: emerging biomarkers. Gastroenterology 2015;149:1204-1225.

27. Dickinson BT, Kisiel J, Ahlquist DA, Grady WM. Molecular markers for colorectal cancer screening. Gut 2015;64:1485-1494.

28. Shah $\mathrm{R}$, Jones $\mathrm{E}$, Vidart $\mathrm{V}$, et al. Biomarkers for early detection of colorectal cancer and polyps: systematic review. Cancer Epidemiol Biomarkers Prev 2014;23:1712-1728.

29. Pepe MS, Alonzo TA. Comparing disease screening tests when true disease status is ascertained only for screen positives. Biostatistics 2001;2:249-260.

30. Muller HM, Oberwalder M, Fiegl H, et al. Methylation changes in faecal DNA: a marker for colorectal cancer screening? Lancet 2004;363:12831285.

31. Huang Z, Li L, Wang J. Hypermethylation of SFRP2 as a potential marker for stool-based detection of colorectal cancer and precancerous lesions. Dig Dis Sci 2007;52:2287-2291.

32. Chen WD, Han ZJ, Skoletsky J, et al. Detection in fecal DNA of colon cancer-specific methylation of the nonexpressed vimentin gene. J Natl Cancer Inst 2005;97:1124-1132.

33. Glockner SC, Dhir M, Yi JM, et al. Methylation of TFPI2 in stool DNA: a potential novel biomarker for the detection of colorectal cancer. Cancer Res 2009;69:4691-4699.

34. Hellebrekers DM, Lentjes MH, van den Bosch SM, et al. GATA4 and GATA5 are potential tumor suppressors and biomarkers in colorectal cancer. Clin Cancer Res 2009;15:3990-3997.

35. Lee BB, Lee EJ, Jung EH, et al. Aberrant methylation of APC, MGMT, RASSF2A, and Wif-1 genes in plasma as a biomarker for early detection of colorectal cancer. Clin Cancer Res 2009;15:6185-6191.

36. Leung WK, To KF, Man EP, et al. Detection of hypermethylated DNA or cyclooxygenase-2 messenger RNA in fecal samples of patients with colorectal cancer or polyps. Am J Gastroenterol 2007;102:1070-1076.

37. Lidgard GP, Domanico MJ, Bruinsma JJ, et al. Clinical performance of an automated stool DNA assay for detection of colorectal neoplasia. Clini Gastroenterol Hepatol 2013;11:1313-1318.

38. Melotte V, Lentjes MH, van den Bosch SM, et al. N-Myc downstream regulated gene 4 (NDRG4): a candidate tumor suppressor gene and potential biomarker for colorectal cancer. J Natl Cancer Inst 2009; 101:916-927.

39. Petko Z, Ghiassi M, Shuber A, et al. Aberrantly methylated CDKN2A, MGMT, and MLH1 in colon polyps and in fecal DNA from patients with colorectal polyps. Clin Cancer Res 2005;11:1203-1209.

40. Wang DR, Tang D. Hypermethylated SFRP2 gene in fecal DNA is a high potential biomarker for colorectal cancer noninvasive screening. World J Gastroenterol 2008;14:524-531.

41. Imperiale TF, Ransohoff DF, Itzkowitz $\mathrm{SH}$, et al. Multitarget stool DNA testing for colorectal-cancer screening. N Engl J Med 2014;370:1287-1297.

42. Wong IH, Lo YM, Zhang J, et al. Detection of aberrant p16 methylation in the plasma and serum of liver cancer patients. Cancer Res 1999;59:71-73.

43. Esteller M, Sanchez-Cespedes M, Rosell R, et al. Detection of aberrant promoter hypermethylation of tumor suppressor genes in serum DNA from non-small cell lung cancer patients. Cancer Res 1999;59:67-70.

44. Silva JM, Dominguez G, Villanueva MJ, et al. Aberrant DNA methylation of the p16INK4a gene in plasma DNA of breast cancer patients. $\mathrm{Br}$ J Cancer 1999;80:1262-1264.

45. Lofton-Day C, Model F, Devos T, et al. DNA methylation biomarkers for blood-based colorectal cancer screening. Clin Chem 2008;54:414-423.

46. Church TR, Wandell M, Lofton-Day C, et al. Prospective evaluation of methylated SEPT9 in plasma for detection of asymptomatic colorectal cancer. Gut 2014;63:317-325. 
Use of Guidelines and Biomarkers for CRC Screening

47. Jin $P$, Kang Q, Wang $X$, et al. Performance of a second-generation methylated SEPT9 test in detecting colorectal neoplasm. J Gastroenterol Hepatol 2015;30:830-833.

48. Ebert MP, Model F, Mooney S, et al. Aristaless-like homeobox-4 gene methylation is a potential marker for colorectal adenocarcinomas. Gastroenterology 2006;131:1418-1430.

49. Leung WK, To KF, Man EP, et al. Quantitative detection of promoter hypermethylation in multiple genes in the serum of patients with colorectal cancer. Am J Gastroenterol 2005;100:2274-2279.
50. Wallner M, Herbst A, Behrens A, et al. Methylation of serum DNA is an independent prognostic marker in colorectal cancer. Clin Cancer Res 2006;12:7347-7352.

51. Herbst A, Rahmig K, Stieber P, et al. Methylation of NEUROG1 in serum is a sensitive marker for the detection of early colorectal cancer. Am J Gastroenterol 2011;106:1110-1118.

52. Li M, Chen WD, Papadopoulos $N$, et al. Sensitive digital quantification of DNA methylation in clinical samples. Nat Biotechnol 2009;27:858-863.

53. Mansour H. Cell-free nucleic acids as noninvasive biomarkers for colorectal cancer detection. Front Genet 2014;5:182. 